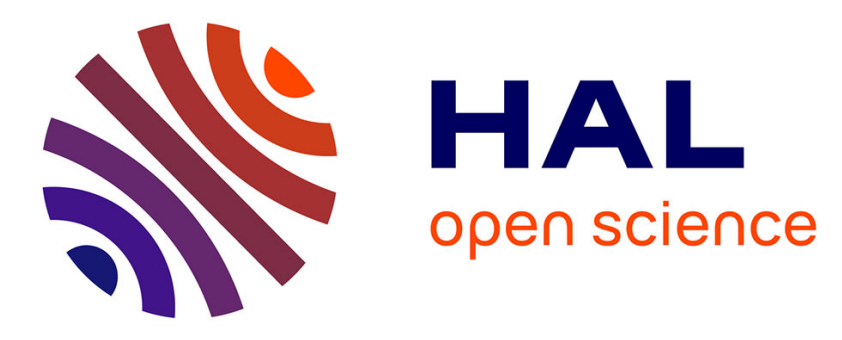

\title{
Modeling malignancies using induced pluripotent stem cells: from chronic myeloid leukemia to hereditary cancers
}

\author{
Ali Turhan, Adlen Foudi, Jin Wook Hwang, Christophe Desterke, Frank \\ Griscelli, Annelise Bennaceur-Griscelli
}

\section{To cite this version:}

Ali Turhan, Adlen Foudi, Jin Wook Hwang, Christophe Desterke, Frank Griscelli, et al.. Modeling malignancies using induced pluripotent stem cells: from chronic myeloid leukemia to hereditary cancers. Experimental Hematology, 2019, 71, pp.61 - 67. 10.1016/j.exphem.2019.01.003 . hal-03486893

\section{HAL Id: hal-03486893 https://hal.science/hal-03486893}

Submitted on 20 Dec 2021

HAL is a multi-disciplinary open access archive for the deposit and dissemination of scientific research documents, whether they are published or not. The documents may come from teaching and research institutions in France or abroad, or from public or private research centers.
L'archive ouverte pluridisciplinaire HAL, est destinée au dépôt et à la diffusion de documents scientifiques de niveau recherche, publiés ou non, émanant des établissements d'enseignement et de recherche français ou étrangers, des laboratoires publics ou privés.

\section{(c) (1) $\$$}

Distributed under a Creative Commons Attribution - NonCommerciall 4.0 International 
MODELING MALIGNANCIES USING INDUCED PLURIPOTENT STEM CELLS: FROM CHRONIC MYELOID LEUKEMIA (CML) TO HEREDITARY CANCERS

\section{A.G. Turhan ${ }^{1,2^{*}}$ A. Foudi ${ }^{3}$, J.W. Hwang ${ }^{1}$, C. Desterke ${ }^{1}$, F. Griscelli ${ }^{1,2,4}$ A. Bennaceur- Griscelli $^{1,2}$}

${ }^{1}$ INSERM UMR-S 935, Université Paris Sud, 94800 Villejuif, France and ESTeam Paris Sud, Université Paris Sud, 94800 Villejuif, France.

${ }^{2}$ INGESTEM National iPSC Infrastructure, 94800 Villejuif, France

${ }^{3}$ ATIP-Avenir INSERM UMR-S 935, Université Paris Sud, 94800 Villejuif

${ }^{4}$ Université Paris Descartes, Faculté Sorbonne Paris Cité, Faculté des Sciences Pharmaceutiques et Biologiques, Paris, France

* Corresponding author 


\section{ABSTRACT}

During the last decade, the possibility of reprogramming malignant cells to a pluripotent state has been achieved in several hematological malignancies, including myeloproliferative neoplasms, myelodysplastic syndromes and chronic myeloid leukemia (CML). It has been shown that it is readily possible to generate iPSC from several types of primary CML cells and generate progenitors and differentiated cells with variable efficiency. Although these experiments have brought some new insights in the understanding of CML pathophysiology, the ultimate goal of generating induced leukemic stem cells (LSC) with long-term multilineage potential has not yet been demonstrated. Experiments underway will determine whether additional signaling events are required to induce the emergence of bona fide LSCs. iPSC modeling offers however the unique possibility to generate pluripotent cells harboring cancer predisposing mutations using patient-derived non-cancerous cells as has been shown in Li-Fraumeni syndrome, BRCA-1 associated breast or RET-mutated medullary thyroid carcinomas. In these conditions, mutated iPSCs can then be used to study the mutational history that precedes the appearance of the malignant transformation and develop novel drug screening strategies. The ability to induce a successful differentiation program towards the tissue in which a given cancer develops or to generate tissue-specific cancer organoids in which the full oncogenic potential can be revealed, remains a major challenge in the field. Similarly, in hematological malignancies a significant hurdle remains owing to the lack of adequate technology to induce the emergence of leukemic stem cells that resemble LSCs, which hinders our ability to study the mechanisms of therapy resistance. 


\section{INTRODUCTION}

The seminal discovery of cell reprogramming in 2006 by Shinya Yamanaka opened up novel and unprecedented perspectives not only in the field of biology in general, but also in all fields of medicine, with almost endless possibilities in terms of therapeutic applications [1]. Although the use induced pluripotent stem cells (iPSC) appeared initially as a technique that would be widely applicable alternatives to embryonic stem cells (ESC), several hurdles remain to be solved for the use of iPSC and their derivatives in regenerative medicine. One of the major problems in this field has been the lack of robust differentiation protocols towards a given tissue with generation of functional cells. Indeed, in the field of hematopoiesis as well as in other fields, the immature status of several types of differentiated cells such as erythrocytes, represents a significant 'bottleneck' for disease modeling strategies. The second concern is the genetic stability of the iPSC lines during several passages with the possibility of occurrence of somatic mutations. The immunogenicity of the cells is also a concern for regenerative medicine approaches. On the other hand, it appeared rapidly that iPSC technology represents a highly spectacular tool of disease modeling in all fields of medicine from neurology to cardiovascular disorders [2]. In the field of cancer, the first hematopoietic malignancy to be studied was the chronic myeloid leukemia (CML) and this field progressed rapidly with development of exciting novel models of myeloproliferative neoplasms [3, 4], myelodysplastic syndromes (MDS) [5], acute myeloid leukemia (AML) [6] and more recently in solid tumors and specifically in hereditary cancers. In MDS, the reprogramming technology allowed the generation of hematopoietic cells from low and high-risk patients and the authors have shown the possibility of testing the effects of the differentiation agent 5-Azacytidine

[7]. We discuss in this review recent developments in the field of CML and hereditary cancers. 


\section{MALIGNANCIES AND PLURIPOTENT STATE: COMMON GROUNDS}

Several lines of evidence suggest that cancer development and cellular reprogramming share many features and signaling pathways. Indeed, since the initial clonal theory of cancer [8] the concepts of cancer development and evolution have evolved considerably. The heterogeneity of cancer was initially thought to be the result of successive mutational events leading to a growth advantage gained by the most aggressive subclones capable of metastatic dissemination. In the tumor microenvironment, all cells were thought to be equal in terms of tumorigenic potential. With the development of stem cell assays and markers, initially in hematopoietic malignancies and later in several types of solid tumors, it has been established that tumors contain functionally distinct populations, a population able to self-renew and another population undergoing differentiation and generating most of the bulk of the tumor. The "cancer stem cell hypothesis" was initially confirmed in the hematopoietic disorders, including myelodysplasia and chronic myeloid leukemia by the demonstration of the clonal nature of these disorders $[9,10]$. The cancer stem cell concept in solid tumors required the development of novel markers and assays which led to their discoveries in glioblastoma [11, 12] colon [13] and breast carcinomas [14]. In particular it has been shown that a small population of cells isolated in human tumors using CD133/ALDH expression or biochemical characteristics (Hoechst efflux) was enriched in cells with self-renewal, proliferation potential and resistance to chemotherapies. The mechanisms of generation of the cancer stem cells (CSC) in a solid tumor has been a subject of a debate as this could be the result of the transforming event taking place in a normal, self-renewing stem cell or alternatively a transforming oncogenic event can induce a de-differentiation potential in a normal differentiated cells which acquire stem cell potential using transcription machinery involved in epithelial mesenchymal transition (EMT) [15]. It has also been shown that the most aggressive cancer cells express embryonic genes [16-18]. Similarly, transcriptome analyses 
performed in this setting have shown that glioblastoma progenitor cells expressed levels of Oc4 and Sox2 similar to those found in human ES cells [19].

The epigenetic resetting that occur during the tumor development is a feature presenting high similarity with the adult cell reprogramming [20]. Conversely, all "Yamanaka" factors (CMyc, Klf4, Sox2, Oct4) have established oncogenic potential and are expressed in several types of cancer cells. Lin28, another reprogramming factor is expressed also in cancer cells and in blast crisis of CML [21]. The use of iPSC could therefore be of major interest to study in vitro, signaling pathways involved in cancer [22]. It appeared however that reprogramming cancer cells are not straightforward due to signaling barriers such as TP53 whose deletion increases the efficiency of reprogramming [23]. If the reprogramming approach is successful, the second important requirement is the ability to induce a differentiation of the tumorderived iPSC towards the tissue in which the primary cancer occurs in order to recapitulate the behavior observed in primary cancer cells. From this regard, a hematopoietic malignancy such as CML was an excellent candidate disorder for cell programming as techniques of hematopoietic differentiation from both murine and human ESC /iPSC have been established. However, generation of definitive hematopoiesis with long-term repopulating activity remains a challenge from both ESC and iPSC.

\section{MODELLING CHRONIC MYELOID LEUKEMIA USING PLURIPOTENT STEM}

\section{CELLS}

CML is a hematopoietic stem cell malignancy arising from a primitive hematopoietic stem cell [24]. The major interest in the CML stem cells during the recent years is related to the fact that despite the major success obtained by the TKI therapies, the most primitive leukemic stem cells are resistant to them [24] leading to relapses upon TKI cessation [25]. Similarly, resistances to TKI therapies occur, leading to progression of the disease on therapy. CML 
stem cells are difficult to isolate and expand and despite some recent cell surface markers such as CD26 [26] there has been no specific biomarkers which have been universally validated in CML [24]. In addition, available in vitro or in vivo mice models do not recapitulate the evolution of human CML, especially with regards to the first stages of the disease and its natural history. To elucidate these questions, we and other turned to model systems to generate ES-based [27, 28] or more recently iPSC-based experimental models. One of the expectations with the use of CML iPSC models was the fact that, as demonstrated in murine ES system, the expression of oncogenic BCR-ABL would lead to the generation of a long-term repopulating ability and therefore a LSC potential which has not been shown so far with the use of neither hESC nor iPSC.

The demonstration of the feasibility of programming CML cells was rapidly shown using even the blast crisis cell line K562 from which BCR-ABL-expressing CD34+ cells, albeit with a very low yield, has been obtained [29]. Further work has confirmed the possibility of cell reprogramming either from total bone marrow, $\mathrm{PBMC}$ or from $\mathrm{Ph} 1+\mathrm{CD} 34+$ cells from patients with CML [30-33]. The analysis of these cell lines has shown the feasibility of generating from the $\mathrm{Ph} 1+\mathrm{iPSC}$, clonogenic progenitors and differentiated hematopoietic cells expressing BCR-ABL. The iPSC lines expressing BCR-ABL were found to be resistant to Imatinib as this might be expected from their pluripotent state but they acquired partial IM sensitivity when induced to differentiation $[32,33]$. The analysis of iPSC-derived CML cells has shown the validity of the novel signaling pathways discovered by this strategy, such as Olfactomedine [34] or ADAM8 [35]. One major question which was a major interest to address with the use of CML iPSC was the possibility to generate leukemic stem cells (LSC) which are known to be highly resistant to TKI [36]. The possibility of obtaining these cells which are difficult to isolate from primary CML marrow, would be of major interest for LSCbased drug targeting purposes. In our center we have reprogrammed leukemic cells from 
several patients with CML at diagnosis with the clinical information with regard to their response to therapies [37]. To analyze the in vitro potential of CML iPSC, we have used CD34+ cells from a patient whose leukemic cells harbored both Ph1 chromosome and a V617F JAK2 mutation [33]. After successful reprogramming and characterization, the "double positive iPSC" were used to determine their hematopoietic potential using CFC and LTC-IC assays combined with their drug sensitivity. Interestingly, we have shown that as compared to human ESC, BCR-ABL /Jak2 mutated iPSC exhibit an increased LTC-IC potential [33]. It is difficult to determine if this potential was due to the presence of both oncogenes or to BCR-ABL alone, but this tool allowed us to validate the use of hematopoietic cells derived from iPSC in drug screening purposes. We have indeed shown in these BCRABL-expressing JAK2-V617F-mutated progenitors that the pimozide, a STAT5 phosphorylation inhibitor, exhibited synergistic inhibitory activity with Imatinib [33]. The possibility of generating from CML iPSC , "LSC-like" cells was also shown by another study [34]. This study also showed that these cells were resistant to Imatinib [34].

Thus, generation of "LSC-like" cells from CML iPSC with either phenotypic markers or LTC-IC generating potential could be shown but there has been so far no data showing that CML-iPSC could generate long-term repopulating LSC in immunodeficient mice. In our studies using several iPSC lines obtained from several patients, we have transplanted into NSG mice, either by intravenous or intrafemoral injection, embryoid bodies (EB) or CD34+ cells derived from CML iPSC but we have not observed a long-term repopulating potential. It appears that the human CML iPSC misses a signaling link which is present in murine pluripotent cells transduced with BCR-ABL. This deceiving result as compared to murine ES cells expressing BCR-ABL $[27,28]$ could be due to the fact that in the murine experimental systems, BCR-ABL expression is under the control of strong promoters as opposed to human 
CML iPSC. The lack of an adequate "niche" allowing the emergence of a primitive LSC potential could also explain these results.

Indeed, it has also been shown that teratomas generated by injection of iPSC in NSG mice can be a permissive microenvironment for spontaneous in vivo generation of hematopoietic cells which could migrate to the bone marrow of NSG mice with a potential to give rise to serial transplantations [38]. It remains to be determined if CML iPSC can acquire LSC potential in the same conditions. Future transcriptome experiments in CML iPSC will also aim to identify a signaling pathways which could enhance this potential. From this regard, it is of interest to notice that the AHR pathway that we identified as a major regulator of malignant progenitor proliferation in CML, could be also a druggable target in CML iPSC to enhance LSC activity [39].

The second important question to validate the use of CML iPSC as disease modeling was the genetic stability of the cell lines as compared to parental primary leukemic cells. Although previous studies have shown that most of the genetic abnormalities observed in iPSC lines were present in the original target cells and disappear during increased passages [40] this question was not addressed in the setting of a neoplastic iPSC. We studied this question by performing a whole genome sequencing (WGS) analysis of our iPSC lines harboring both Ph1 chromosome and V617F JAK2 mutation as compared to cryopreserved primary leukemic cells of the same patient. This analysis showed a highly preserved genomic structure during and after reprogramming with very few additional mutations, suggesting excellent "genocopy" of the primary leukemic cells during iPSC generation [33]. The demonstration of the adequate "capture" of genomic alterations present in primary leukemic cells upon cellular reprogramming was an important step forward in order to validate the possibility of using the iPSC-derived CML models with regard to the CML pathophysiology and for future studies. 
Figure 1 shows some achievements and summarizes future challenges in the field of CML using iPSC technology.

\section{MODELING HEREDITARY CANCERS USING IPSC: FROM PREDISPOSITION TO "DISEASE IN A DISH"}

As opposed to hematopoietic malignancies and in particular CML and other myeloproliferative neoplasms, reprogramming cancer cells to pluripotency has proven to be difficult. Early experiments of nuclear transfer performed before cell reprogramming technologies have shown that it was not possible to reprogram nuclei of several tumors (breast, lymphoma) to pluripotency except for melanoma from which a pluripotent stem cell line could be developed by nuclear transfer [41]. These early results suggested that at least in some cancer cells, the presence of an established malignant phenotype was compatible with genome reprogramming. The rarity of this event could be due to the presence of complex abnormalities present in these cells. The use of iPSC technology has confirmed later the relative inefficiency of cell programming in malignant cells from solid tumors in general. In the case of pancreas cancer, an iPSC line from a pancreas adenocarcinoma cell could be obtained with a very low efficiency using a DOX-inducible gene transfer system [42]. However, this iPSC cell line required continuously the expression of pluripotency factor expression (as it required the addition of Dox in the cell culture) and probably does not reflect a true 'pancreas cancer iPSC' [42]. The major inefficiency of reprogramming of a bona fide cancer cell to pluripotency could also be related to the fact that cancer cells could require additional reprogramming factors as compared to normal cells as it has been shown in glioblastoma [43]. The ability to reprogram solid tumors for identification of early stages of oncogenesis remains a challenge that will require further efforts underway in several laboratories. The difficulty of obtaining "cancer iPSC" from transformed cells stimulated 
efforts to generate in vitro cancer models by using gene editing technology from normal iPSC [44]. Figure 2B summarizes different strategies which can be used to generate a "cancer iPSC" in vitro but the possibility of recreating a tumor phenotype reminiscent of human cancer remains to be confirmed in future studies.

As opposed to sporadic cancers that accumulate genomic abnormalities leading to their discovery, hereditary cancers are characterized by long lasting latency in the presence of a germline genomic event known to predispose to the occurrence of cancer. In this context, cell programming represents a fascinating modeling tool as a germline oncogenic mutation or deletion of a tumor suppressor gene is already present in a otherwise healthy donor tissues. It can allow to analyze the cell of origin of initial neoplastic transformation and the tissue specificity of a given cancer, by the theoretically unlimited differentiation potential of pluripotent cells. Mutations or deletion in genes such as BRCA1 and TP53 represent one of the earliest events in familial breast cancers and Li-Fraumeni syndromes, respectively. Although in these disorders, the classical 'second hit' concept refers to the loss of a second allele, the signaling events induced in the oncogenic transformation are probably much more complex, implicating epigenetic abnormalities. Similarly, the tissue specificity of the cancer predisposition remains poorly elucidated. One of the prerequisites of the model is the ability to induce from iPSC, a tissue or organoid close to the organ in which the cancer arises in vivo. The possibility of modeling Li-Fraumeni syndrome using iPSC has been demonstrated using patient-specific iPSC with osteosarcoma phenotype generated after osteoblastic differentiation [45]. It has been shown that LFS iPSC-derived osteoblasts present features of LFS osteosarcoma both phenotypically and using gene expression analyses [45]. In iPSC-derived osteosarcoma cells there was a major down-regulation of H19 gene and its overexpression reverted the tumorigenic potential of osteosarcoma cells [45]. These experiments were clearly the first showing the major interest of cell programming in the setting of a hereditary cancer. 
BRCA1-mutated breast cancer represents another attractive target for cell programming strategies. BRCA1, "guardian of genome integrity" plays a critical role in DNA repair and its alteration by mutations or deletion is associated with the predisposition of the occurrence of familial breast and ovarian cancers. Similar to Li-Fraumeni syndrome, the possibility of obtaining a BRCA1-altered iPSC offers the possibility of studying secondary genetic events associated with the BRCA1 abnormalities. It has been shown for instance, the functional abrogation of the second allele of BRCA1 could be, albeit infrequently, achieved by an epigenetic mechanism [46]. The availability of the BRCA1 iPSC could allow to test in vitro and in vivo the phenotypic abnormalities observed by modification of gene expression or genome editing. The possibility of generating BRCA1-IPSc has been shown by Soyombo and colleagues [47] and our group [48]. This unique BRCA-deleted iPSC from a patient with exon 17 deletion all features of pluripotency using in vitro and in vivo assays with no evidence of malignant tumor in teratomas generated in NSG mice [48].

One other interesting target for cell programming in the case of cancer predisposition is the Multiple endocrine neoplasia 2A (MEN2A) syndrome due to germline mutation of c-RET and including essentially medullary thyroid cancer (MTC) and pheochromocytoma [49]. C-RET encodes for a transmembrane tyrosine kinase and its activation occurs through heterodimerization with the glial derived neurotrophic factor (GDNF) receptor in the presence of the ligands of the GDNF family. The oncogenic mutant form has increased TK activity leading to cell proliferation in the absence of the ligand. The disease can vary in severity as a function of the mutation, the most aggressive disease being due to the c-RET mutant M918T. We had the opportunity to generate iPSC using PBMC of three patients with various c-RET mutations followed by genomic and functional analyses [50, 51]. All three iPSC lines with RET mutations expressed pluripotency markers and led to teratomas formation with trilineage differentiation when injected into immunodeficient NSG mice. Although we could not 
observe any neoplastic phenotype either in vitro or in vivo, we were able to derive isogeneic corrected iPSC (RET-Y634C) in the case of a "high-risk" C634Y mutated iPSC using CRISPR/CAS9 technology [50]. Both mutated and corrected iPSC lines were subjected to exome sequencing to evaluate potential off-target events which were minimal [50]. Interestingly, integrative differential gene expression analysis of RET-C634Y, RET-Y634C and RET-wild type iPSCs revealed activation of the Early Growth Response 1 (EGR1) transcriptional program specifically in RET-C634Y mutated iPSC, which had been previously suggested based on studies using cancer cell lines.

The bioinformatics analysis revealed that EGR1 elicited a transcriptional program specifically in iPSC harboring oncogenic RET, in two different RET-mutated iPSC lines [50]. Although we could not generate a terminally differentiated calcitonin-secreting $\mathrm{C}$ cells, these findings strongly confirmed the feasibility of target discovery strategies using RET-mutated iPSC and showed as proof of concept that iPSC can be used as a potent platform in hereditary cancers. One important additional advantage using iPSC in the context of hereditary neoplasms is the ability to develop in vitro 3D organoids for instance using iPSC derived from patients with BRCA1-mutated breast or c-MET-mutated renal carcinomas. The possibility of modeling these cancers using iPSC technology offers novel exciting perspectives as the initial genomic abnormality is carried at the pluripotent stage but also during tissue specification and cellular differentiation. Recent data showed the feasibility of the modeling the familial adenomatous polyposis (FAP) characterized by germline mutation of WNT signaling pathway using iPSCderived colonic organoids which could then be used as a drug screening platform [52].

\section{PERSPECTIVES}

The possibility of modifying the cell fate by reprogramming changed forever the field of cell biology with potential major therapeutic perspectives in all fields of medicine. Recent 
development of iPSC-derived organoid technology combined with CRISPR-CAS9 revolution allows now unprecedented innovations in the field of disease modeling with generation of cancer models from both hematological and non-hematological malignancies. In the field of hematological malignancies, one important challenge remains the possibility of generating a "true" LSC with long-term repopulating potential, both in CML and other myeloproliferative and myelodysplastic disorders. In the field of solid tumors, iPSC technology can be of major interest in modeling hereditary cancers and progress will be commensurate to that of organoid technology. Reprogramming a cancer cell with a fully transformed phenotype is still a challenge but will certainly be possible with the improved understanding of epigenetics of the cancer cell and will probably open up novel perspectives for future cancer therapy approaches.

\section{REFERENCES}

[1] Takahashi K, Yamanaka S. Induction of pluripotent stem cells from mouse embryonic and adult fibroblast cultures by defined factors. Cell. 2006;126:663-676.

[2] Inoue H, Nagata N, Kurokawa H, Yamanaka S. iPS cells: a game changer for future medicine. EMBO J. 2014;33:409-417.

[3] Hosoi M, Kumano K, Taoka K, et al. Generation of induced pluripotent stem cells derived from primary and secondary myelofibrosis patient samples. Exp Hematol. 2014;42:816-825.

[4] Tasian SK, Casas JA, Posocco D, et al. Mutation-specific signaling profiles and kinase inhibitor sensitivities of juvenile myelomonocytic leukemia revealed by induced pluripotent stem cells. Leukemia. 2018.

[5] Kotini AG, Chang CJ, Boussaad I, et al. Functional analysis of a chromosomal deletion associated with myelodysplastic syndromes using isogenic human induced pluripotent stem cells. Nat Biotechnol. 2015;33:646-655.

[6] Chao MP, Gentles AJ, Chatterjee S, et al. Human AML-iPSCs Reacquire Leukemic Properties after Differentiation and Model Clonal Variation of Disease. Cell Stem Cell. 2017;20:329-344.e327.

[7] Kotini AG, Chang CJ, Chow A, et al. Stage-Specific Human Induced Pluripotent Stem Cells Map the Progression of Myeloid Transformation to Transplantable Leukemia. Cell Stem Cell. 2017;20:315-328.e317.

[8] Nowell PC. The clonal evolution of tumor cell populations. Science. 1976;194:2328. 
[9] Janssen JW, Buschle M, Layton M, et al. Clonal analysis of myelodysplastic syndromes: evidence of multipotent stem cell origin. Blood. 1989;73:248-254.

[10] Fialkow PJ, Jacobson RJ, Papayannopoulou T. Chronic myelocytic leukemia: clonal origin in a stem cell common to the granulocyte, erythrocyte, platelet and monocyte/macrophage. Am J Med. 1977;63:125-130.

[11] Singh SK, Hawkins C, Clarke ID, et al. Identification of human brain tumour initiating cells. Nature. 2004;432:396-401.

[12] Piccirillo SG, Reynolds BA, Zanetti N, et al. Bone morphogenetic proteins inhibit the tumorigenic potential of human brain tumour-initiating cells. Nature. 2006;444:761765.

[13] O'Brien CA, Pollett A, Gallinger S, Dick JE. A human colon cancer cell capable of initiating tumour growth in immunodeficient mice. Nature. 2007;445:106-110.

[14] Al-Hajj M, Wicha MS, Benito-Hernandez A, Morrison SJ, Clarke MF. Prospective identification of tumorigenic breast cancer cells. Proc Natl Acad Sci U S A. 2003;100:3983-3988.

[15] Tam WL, Weinberg RA. The epigenetics of epithelial-mesenchymal plasticity in cancer. Nat Med. 2013;19:1438-1449.

[16] Wong DJ, Liu H, Ridky TW, Cassarino D, Segal E, Chang HY. Module map of stem cell genes guides creation of epithelial cancer stem cells. Cell Stem Cell. 2008;2:333-344.

[17] Ben-Porath I, Thomson MW, Carey VJ, et al. An embryonic stem cell-like gene expression signature in poorly differentiated aggressive human tumors. Nat Genet. 2008;40:499-507.

[18] Bass AJ, Watanabe $\mathrm{H}$, Mermel $\mathrm{CH}$, et al. SOX2 is an amplified lineage-survival oncogene in lung and esophageal squamous cell carcinomas. Nat Genet. 2009;41:12381242.

[19] Balbous A, Cortes U, Guilloteau K, et al. A mesenchymal glioma stem cell profile is related to clinical outcome. Oncogenesis. 2014;3:e91.

[20] Kim J, Orkin SH. Embryonic stem cell-specific signatures in cancer: insights into genomic regulatory networks and implications for medicine. Genome Med. 2011;3:75.

[21] Viswanathan SR, Powers JT, Einhorn W, et al. Lin28 promotes transformation and is associated with advanced human malignancies. Nat Genet. 2009;41:843-848.

[22] Shibue T, Weinberg RA. EMT, CSCs, and drug resistance: the mechanistic link and clinical implications. Nat Rev Clin Oncol. 2017;14:611-629.

[23] Hong H, Takahashi K, Ichisaka T, et al. Suppression of induced pluripotent stem cell generation by the p53-p21 pathway. Nature. 2009;460:1132-1135.

[24] Chomel JC, Turhan AG. Chronic myeloid leukemia stem cells in the era of targeted therapies: resistance, persistence and long-term dormancy. Oncotarget. 2011;2:713727.

[25] Mahon FX, Réa D, Guilhot J, et al. Discontinuation of imatinib in patients with chronic myeloid leukaemia who have maintained complete molecular remission for at least 2 years: the prospective, multicentre Stop Imatinib (STIM) trial. Lancet Oncol. 2010;11:1029-1035.

[26] Herrmann H, Sadovnik I, Cerny-Reiterer S, et al. Dipeptidylpeptidase IV (CD26) defines leukemic stem cells (LSC) in chronic myeloid leukemia. Blood. 2014;123:39513962.

[27] Perlingeiro RC, Kyba M, Daley GQ. Clonal analysis of differentiating embryonic stem cells reveals a hematopoietic progenitor with primitive erythroid and adult lymphoid-myeloid potential. Development. 2001;128:4597-4604. 
[28] Melkus M, Bennaceur-Griscelli A, Valogne Y, et al. Biological effects of T315Imutated BCR-ABL in an embryonic stem cell-derived hematopoiesis model. Exp Hematol. 2013;41:335-345.e333.

[29] Carette JE, Pruszak J, Varadarajan M, et al. Generation of iPSCs from cultured human malignant cells. Blood. 2010;115:4039-4042.

[30] $\mathrm{Hu} \mathrm{K}, \mathrm{Yu} \mathrm{J}$, Suknuntha K, et al. Efficient generation of transgene-free induced pluripotent stem cells from normal and neoplastic bone marrow and cord blood mononuclear cells. Blood. 2011;117:e109-119.

[31] Kumano K, Arai S, Hosoi M, et al. Generation of induced pluripotent stem cells from primary chronic myelogenous leukemia patient samples. Blood. 2012;119:62346242.

[32] Bedel A, Pasquet JM, Lippert E, et al. Variable behavior of iPSCs derived from CML patients for response to TKI and hematopoietic differentiation. PLoS One. 2013;8:e71596.

[33] Sloma I, Mitjavila-Garcia MT, Feraud 0, et al. Whole-genome analysis reveals unexpected dynamics of mutant subclone development in a patient with JAK2-V617Fpositive chronic myeloid leukemia. Exp Hematol. 2017;53:48-58.

[34] Suknuntha K, Ishii Y, Tao L, et al. Discovery of survival factor for primitive chronic myeloid leukemia cells using induced pluripotent stem cells. Stem Cell Res. 2015;15:678-693.

[35] Miyauchi M, Koya J, Arai S, et al. ADAM8 Is an Antigen of Tyrosine Kinase Inhibitor-Resistant Chronic Myeloid Leukemia Cells Identified by Patient-Derived Induced Pluripotent Stem Cells. Stem Cell Reports. 2018;10:1115-1130.

[36] Graham SM, Jørgensen HG, Allan E, et al. Primitive, quiescent, Philadelphiapositive stem cells from patients with chronic myeloid leukemia are insensitive to STI571 in vitro. Blood. 2002;99:319-325.

[37] Telliam G, Féraud O, Griscelli F, et al. Generation of an induced pluripotent stem cell line from a patient with chronic myeloid leukemia (CML) resistant to targeted therapies. Stem Cell Res. 2016;17:235-237.

[38] Suzuki N, Yamazaki S, Yamaguchi T, et al. Generation of engraftable hematopoietic stem cells from induced pluripotent stem cells by way of teratoma formation. Mol Ther. 2013;21:1424-1431.

[39] Gentil M, Hugues P, Desterke C, et al. Aryl hydrocarbon receptor (AHR) is a novel druggable pathway controlling malignant progenitor proliferation in chronic myeloid leukemia (CML). Plos One. 2018;13.

[40] Hussein SM, Batada NN, Vuoristo S, et al. Copy number variation and selection during reprogramming to pluripotency. Nature. 2011;471:58-62.

[41] Hochedlinger $\mathrm{K}$, Blelloch $\mathrm{R}$, Brennan $\mathrm{C}$, et al. Reprogramming of a melanoma genome by nuclear transplantation. Genes Dev. 2004;18:1875-1885.

[42] Kim J, Hoffman JP, Alpaugh RK, et al. An iPSC line from human pancreatic ductal adenocarcinoma undergoes early to invasive stages of pancreatic cancer progression. Cell Rep. 2013;3:2088-2099.

[43] Suvà ML, Rheinbay E, Gillespie SM, et al. Reconstructing and reprogramming the tumor-propagating potential of glioblastoma stem-like cells. Cell. 2014;157:580-594.

[44] Sancho-Martinez I, Nivet E, Xia Y, et al. Establishment of human iPSC-based models for the study and targeting of glioma initiating cells. Nat Commun. 2016;7:10743.

[45] Lee DF, Su J, Kim HS, et al. Modeling familial cancer with induced pluripotent stem cells. Cell. 2015;161:240-254. 
[46] Dworkin AM, Spearman AD, Tseng SY, Sweet K, Toland AE. Methylation not a frequent "second hit" in tumors with germline BRCA mutations. Fam Cancer. 2009;8:339-346.

[47] Soyombo AA, Wu Y, Kolski L, et al. Analysis of induced pluripotent stem cells from a BRCA1 mutant family. Stem Cell Reports. 2013;1:336-349.

[48] Griscelli F, Oudrhiri N, Feraud O, et al. Generation of induced pluripotent stem cell (iPSC) line from a patient with triple negative breast cancer with hereditary exon 17 deletion of BRCA1 gene. Stem Cell Res. 2017;24:135-138.

[49] Wells SA, Pacini F, Robinson BG, Santoro M. Multiple endocrine neoplasia type 2 and familial medullary thyroid carcinoma: an update. J Clin Endocrinol Metab. 2013;98:3149-3164.

[50] Hadoux J, Desterke C, Féraud 0, et al. Transcriptional landscape of a RET. Stem Cell Res. 2018;26:8-16.

[51] Bennaceur-Griscelli A, Hadoux J, Féraud 0, et al. Generation of an induced pluripotent stem cell line from a patient with hereditary multiple endocrine neoplasia 2B (MEN2B) syndrome with "highest risk" RET mutation. Stem Cell Res. 2017;23:154157.

[52] Crespo M, Vilar E, Tsai SY, et al. Colonic organoids derived from human induced pluripotent stem cells for modeling colorectal cancer and drug testing. Nat Med. 2017;23:878-884.

\section{FIGURE LEGENDS}

FIGURE 1. Schematic representation of some achievements for disease modeling in CML using patient-derived IPSC. Several important findings have been reported with regard to CML pathophysiology but the major "bottleneck" remains the inability of generating longterm LSC. POC: proof of concept.

FIGURE 2. Schematic representation of different strategies used in cancer modeling using IPSC technology. Reprogramming of bona fide cancer cells to a pluripotent phenotype has proven to be difficult, probably due to genetic and epigenetic modifications accumulated in cancer cells (Figure 2A). These difficulties could be experimentally circumvented using genome editing of normal iPSC, in order to induce a malignant phenotype, such as reported in a model of experimental glioma ${ }^{43}$ (Figure 2B). In the context of cancer predisposition syndromes (Figure 2C), cell reprogramming of a healthy tissue carrying an oncogenic 
mutation has been possible in several types of hereditary cancers ${ }^{44,46-49}$. These cells can then be used to perform genome editing to determine the effects of gene correction ${ }^{48}$ or to generate organoids in which the genomic events required for full transformation can be tested. 


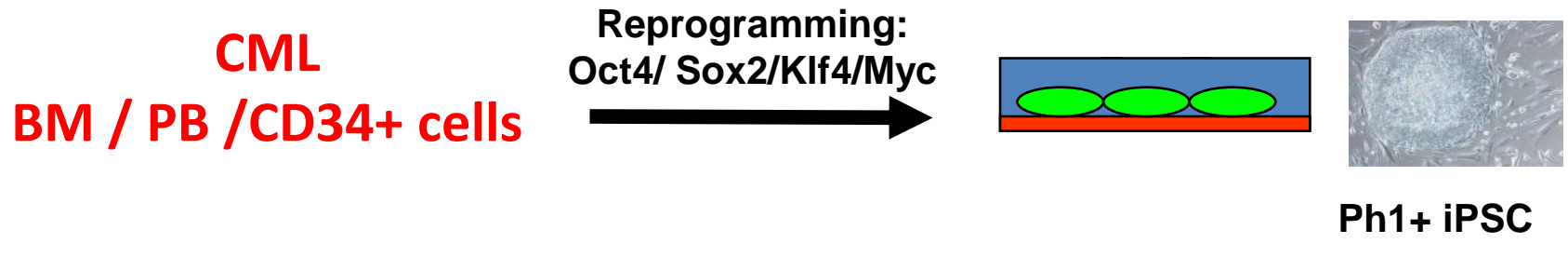

\section{Recent highlights}

-Highly efficient CML iPSC generation protocols ${ }^{28-32,36}$

-Short term-hematopoiesis from CML iPSC possible en-32 $^{28}$

-CML iPSC: capture of primary $\mathrm{CML}_{\text {genome }}{ }^{32}$

-CFC assays : TKI sensitivity appears in clonogenic cells ${ }^{30-33}$

-Discovery of novel signaling pathways ${ }^{33,34}$

\section{IPSC-derived leukemic hematopoiesis}

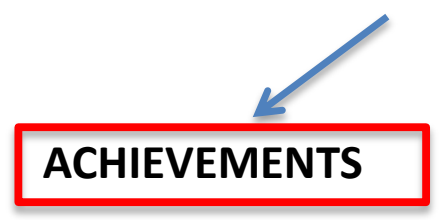

In Vitro " LSC like ${ }^{33}$ LTC-IC-derived CFC ${ }^{32}$

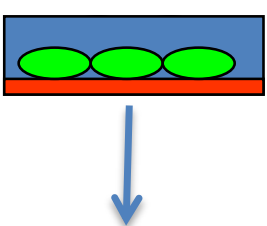

Transcriptome Exome Sequencing WGS

POC Drug Screen ${ }^{32}$

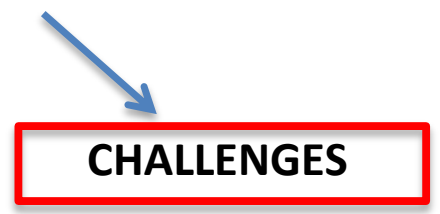

In Vivo Long-term

LSC generation in NSG Mice
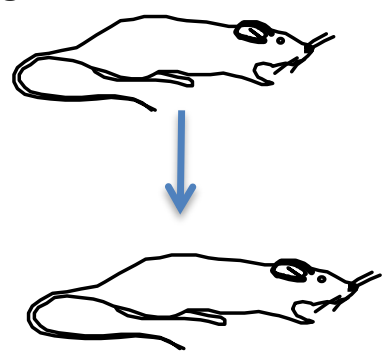

Human CD45+ / CD34+ cells ?

\section{Future Challenges}

- Induction of LSC potential ? > Not demonstrated

- Amplification of Ph1+ LSC ?

- Mechanisms of genetic instability?

- Mechanisms of blast crisis ? 
FIGURE 2

\section{Cancer Cell}

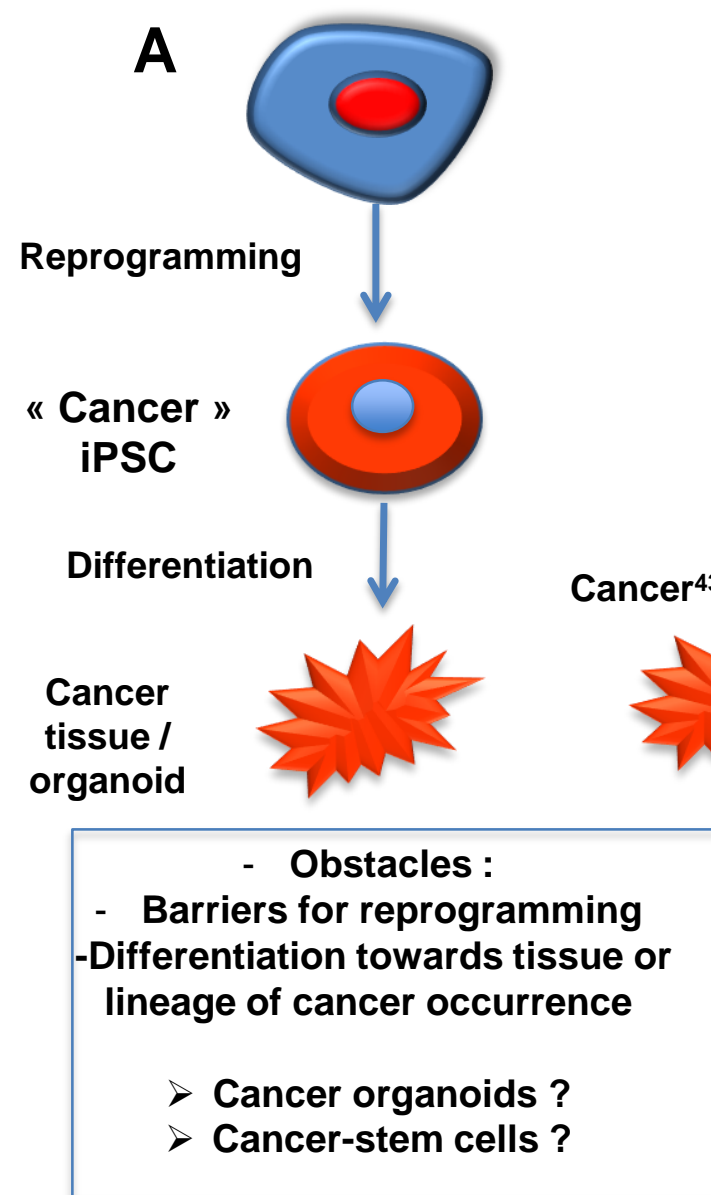

« Normal » iPSC

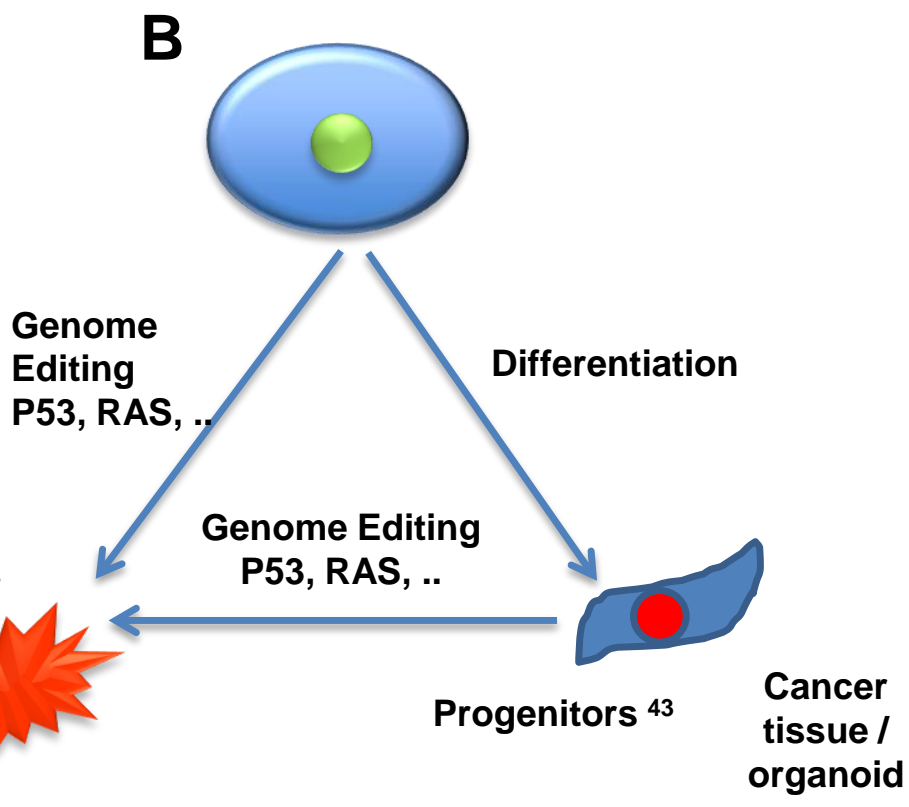

Somatic Cell (Hereditary Cancer)

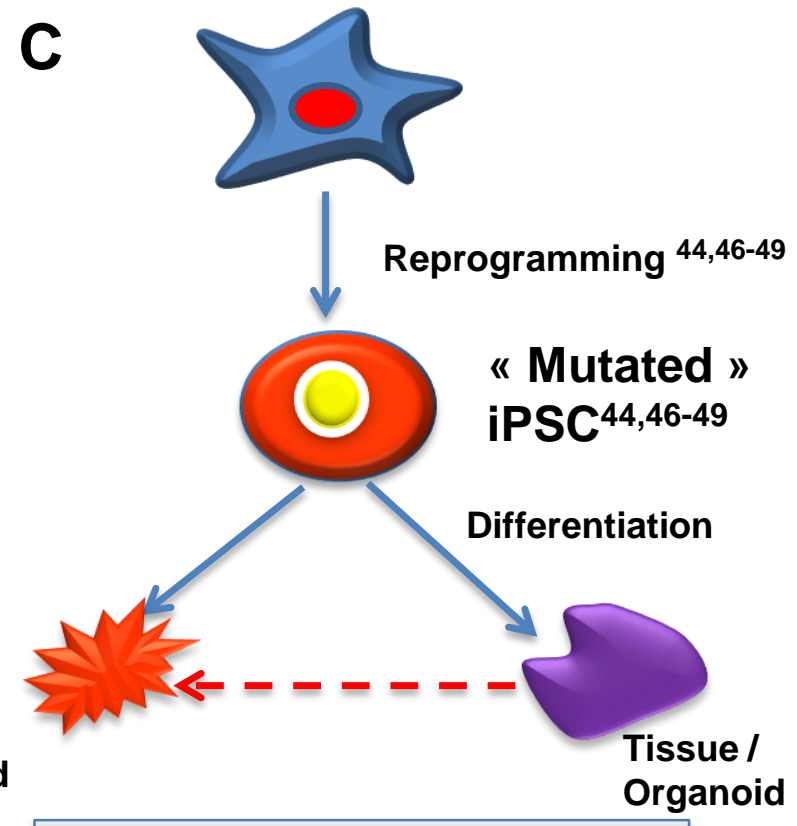

Generation of a Glioma model 43 Application to other cancers? Generation of cancer organoid or tissue?
Cancer Organoid?

Genome Editing ${ }^{48}$

Transcriptome from organoids 48

Drug screening? 